\title{
Probabilistic assessment of nectar requirements for nectar-foraging honey bees
}

\author{
Sara RODNEY ${ }^{1}$, Vincent J. KRAMER ${ }^{2}$ \\ ${ }^{1}$ Intrinsik Corp, 208-2120 Robertson Rd., Ottawa, ON K2H 5Z1, Canada \\ ${ }^{2}$ Corteva Agriscience, 9330 Zionsville Rd., Indianapolis, IN 46268, USA
}

Received 6 December 2018 - Revised 24 July 2019 - Accepted 12 September 2019

\begin{abstract}
Recent concerns regarding potential effects of pesticides on pollinators have prompted regulatory agencies to estimate dietary ingestion rates for honey bees (Apis mellifera). The task is difficult because of the complex caste and food storage systems in honey bee colonies. Considerable data on the nutrition and energetics of honey bees have recently been collated. These data were used to parameterize a probabilistic model estimating nectar requirements of nectar foragers. Median estimates were more than $6 \times$ lower than the recommended median value from the North American government agencies, of $292 \mathrm{mg}$ nectar/bee/day. The distribution of estimates had much greater variability than those of the agencies. The differences are due primarily to the disparate assumptions regarding how much time nectar foragers spend flying and foraging. Risk assessors considering honey bee dietary exposure should take account of current and emerging data providing insight into nectar forager dietary requirements, foraging activity, and feeding behavior.
\end{abstract}

probabilistic / modeling / nectar / Apis mellifera / honey bee / energetics / foraging

\section{INTRODUCTION}

Dietary exposure is typically estimated by multiplying residues in food by an assumed food ingestion rate. This has been a long-standing approach for vertebrates in regulatory risk assessment and is more recently found in regulatory guidance for pollinators, including honey bees (Apis mellifera; EFSA 2013; EPA, PMRA and CDPR 2014). In 2014, the US Environmental Protection Agency (EPA), in collaboration with the Canadian Pest Management Regulatory Agency (PMRA) and the California Department of Pesticide Regulation (CDPR), released a guidance document for

Electronic supplementary material The online version of this article (https://doi.org/10.1007/s13592-019-00693-w) contains supplementary material, which is available to authorized users.

Corresponding author: V. Kramer, vince.kramer@corteva.com

Manuscript editor: James Nieh assessing pesticide risk to bees (EPA, PMRA and CDPR 2014). The protection goals specified in the guidance are to ensure bee biodiversity, provision of pollination services, and production of hive products. At the individual level of biological organization, the measurement endpoints are worker survival, larval emergence, and queen fecundity/reproduction. Nectar foragers are expected to have the highest dietary exposure of all honey bee castes because of their elevated energy requirements during flight (EPA, PMRA and CDPR 2012; 2014). To assess dietary exposure of nectar foragers, the Agencies modeled daily nectar requirement rates. This analysis was presented in detail in a White Paper that preceded the guidance document (EPA, PMRA and CDPR 2012). Pollen requirements were also estimated, but as discussed in the White Paper and in Rodney and Purdy (this issue), pollen is not a significant food source for bees of nectarforaging age. The Agencies' model was probabilistic and included input variables accounting for the amount and duration of flying trips and the amount of sugar 
required to fly and rest. The Agencies selected the median estimate from the output distribution for nectar ingestion rate (i.e., $292 \mathrm{mg}$ nectar/bee/day) as the basis for estimating exposure in screening-level assessments. In their proposed exposure assessment, conservatively, it is assumed that there is no degradation or dissipation of the pesticide between application and ingestion. Available data from Harano and Nakamura (2016) demonstrate that foragers acquire fuel for foraging from processed nectar in the hive in advance of departure.

The Agencies' model was based on sparse input data and was not subject to a sensitivity analysis. A comprehensive review of the literature was undertaken to characterize the available data relating to the nectar requirements of nectar foragers (Rodney and Purdy (this issue)). The objective of the analysis described in this paper is to compare ingestion rate estimates derived in the Agencies' original modeling with those from an alternative model (RP model) employing data compiled by Rodney and Purdy (this issue) and with the Agencies' model parameterized with data compiled by Rodney and Purdy (this issue). Additionally, the sensitivity of the models was assessed and the effect of potential correlations in the Agencies' model was explored.

\section{METHODS}

\subsection{Agencies' model}

The model presented in the White Paper for nectar consumption rate of nectar foragers is a function of trips per day $(T)$, trip duration $\left(D_{i}\right)$, sugar required for flight $\left(S_{\mathrm{F}}\right)$, sugar required to meet resting metabolic rate $\left(\mathrm{S}_{\mathrm{R}}\right)$, and sugar content of nectar $\left(P_{i}\right.$ and $\left.P_{\text {ave }}\right)$, as presented below:

$$
\begin{aligned}
D_{\text {nectar }}= & S_{\mathrm{F}} * \sum_{i=0}^{T}\left(\frac{D_{i} * F_{i}}{P_{i}}\right) \\
& +\frac{S_{\mathrm{R}} *\left[24-\left(\sum_{i=0}^{T} D_{i} * F_{i}\right)\right]}{P_{\text {ave }}}
\end{aligned}
$$

where

$D_{\text {nectar }}$ Nectar requirement (mg nectar/bee/day)

$T \quad$ Number of trips per day
$S_{\mathrm{F}} \quad$ Sugar required for flight $(\mathrm{mg} / \mathrm{h})$

$S_{\mathrm{R}} \quad$ Sugar required to meet resting metabolic rate $(\mathrm{mg} / \mathrm{h})$

$F_{i} \quad$ Fraction of time spent flying during trip $i$

$D_{i} \quad$ Duration of foraging trip $i(\mathrm{~h})$

$P_{i} \quad$ Sugar content of collected nectar during trip $i$

$P_{\text {ave }} \quad$ Average sugar content of nectar

The Agencies used a probabilistic version of this model to simulate 10,000 nectar-foraging bees. For five of the seven parameters listed above, the Agencies assumed continuous distributions. For $S_{\mathrm{R}}$ and $P_{\text {ave }}$, point estimates were used (Table I). The model described by the Agencies in the White Paper was reproduced in MS Excel ${ }^{\circledR} 2016$ with Oracle ${ }^{\circledR}$ Crystal Ball (Release 11.1.2.4.600), hereafter referred to as the RA model (Reproduction of the Agencies' model).

In the RA model, the number of trips per day, $T$, was set up as a single assumption using the inputs from Table I. Subsequently, for each potential trip $i=1$ to $150, D_{i}, F_{i}$, and $P_{i}$ were selected from the continuous distributions specified in Table I. For each of these trips, $D_{i} * F_{i}$ (the time on the trip spent flying in hours) was calculated. The resulting value was divided by $P_{i}\left(\left[D_{i}{ }^{*} F_{i}\right] /\right.$ $\left.P_{i}\right)$.

The selected number of trips, $T$, was then used in each trial to determine how many of the trips simulated would be used in each trial to estimate a nectar consumption rate. The Agencies did not specify how the continuous lognormal estimates were used to estimate whole numbers of trips, $i=$ 1 to 150 . This can be done by rounding the sampled value to the nearest whole number, which is what we did.

\subsection{Revised input assumptions for the Agencies' model}

Subsequently, we ran the RA model with revised input assumptions based on data collated in Rodney and Purdy (this issue). The RA model with revised inputs is referred to herein as the RARI (Reproduction of Agencies' with Revised Inputs) model.

For each of the revised input assumptions, data were considered for quantitative use if they met the 


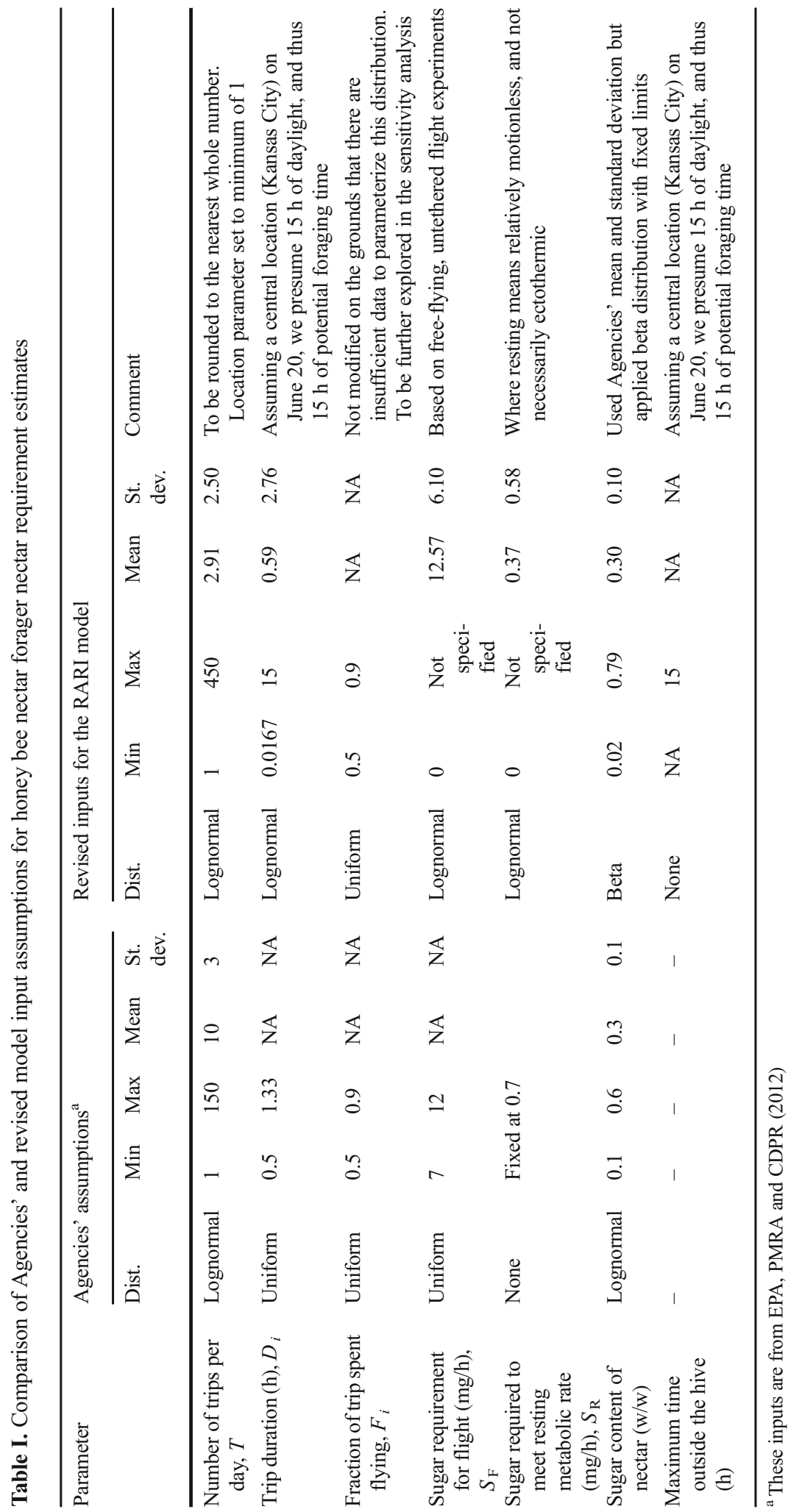


following quality criteria: (1) sample size was reported, (2) sample mean and/or sample standard error, standard deviation, or variance were reported, (3) there was no application of a pesticide, and (4) there were no significant treatmentrelated effects if a stressor (other than a pesticide) was applied to a sample group. When results for metabolism were presented at multiple air temperatures, we selected those closest to the average air temperature in June of approximately $22{ }^{\circ} \mathrm{C}$ in the central USA. Also, with respect to flights (frequency and duration), we only considered data from bees foraging on plants, as opposed to prepared sugar solutions, to avoid using unrealistic observations in the modeling. All data used to generate model inputs are provided in Online Resource 1.

Weighted means were calculated based on reported means and sample sizes from available studies. That is, reported means were weighted according to the corresponding sample size. The corresponding pooled sample variance calculations were based on reported sample sizes, means, and standard deviations or standard errors (Headrick 2010; Table I).

\subsubsection{Trip inputs, $T$ and $D_{i}$}

The number of trips taken in a given day by a nectar forager varies among subspecies, colonies, individuals, and temporally for each individual depending on the age and health of the forager, colony needs, available resources, and weather conditions (reviewed in Rodney and Purdy, this issue). The most comprehensive data currently available for the frequency of trips taken by workers is based on radio-frequency identification (RFID) tracking of individual bees over extended periods of time, typically days to weeks (Thompson et al. 2016; Dosselli et al. 2016). The results are presented as averages of individual bees over numerous flying days. Thus, these results, as well as the less robust datasets from other published studies investigating trips per day, do not include day-to-day variability in the number of trips taken by an individual. Based on the results of $\mathrm{He}$ et al. (2013), we generally expect to see an increase in individual foraging time with age.

Bees avoid foraging on days of inclement weather. As reviewed in Rodney and Purdy (this issue), not all bees of foraging age forage every day, even if the weather conditions are favorable. A conservative approach is to only consider bees that are foraging when estimating the number of trips taken per day by bees of foraging age (i.e., the minimum number of trips per day is one), as was done by the Agencies.

Based on quantitative data from five studies, a mean of $2.91 \pm 2.50$ trips per day was calculated for $T$ ( $n=2713$ for sample mean, $n=2257$ for sample standard deviation; Heberle 1914; Thom et al. 2000; Lach et al. 2015; Dosselli et al. 2016; Thompson et al. 2016; Online Resource 1, Table S1-1 Data used to estimate the distribution parameters for the number of trips per day $(T)$ taken by honey bee nectar foragers in the RARI model). Other studies did not report sample size or variance but did report mean flights per bee per day of between 7 and 13.5 trips (Park 1922; Lundie 1925) with single day maxima of 17 and 24 , for bees foraging on white clover in Iowa (Park 1922). These results were based on counts made by observers, which may be more prone to error than RFID tracking.

The data used to estimate the number of trips per day suggest a right skew. A discrete distribution, such as Poisson, may be considered a more appropriate distribution for count data. In this case, however, the variance indicates overdispersion if the Poisson distribution is assumed. Thus, we assumed a lognormal distribution, which is consistent with the Agencies' approach, with a location parameter (minimum) of 1 . The selected number of trips was rounded to a whole number in the simulations. We assumed (1) $15 \mathrm{~h}$ of daylight (approximately the longest day in the center of the contiguous USA) and (2) arbitrary minimum trip and turnaround times of 1 min and accordingly set a theoretical maximum number of round trips to 450 . We note here that the Agencies' upper limit of 150 trips per day was based on a value presented in Winston (1987) that originated from Butler et al. (1943). The latter reported that two bees foraging on sugar solution made approximately 150 trips by early evening. Most of the 
literature sources for trip duration data, $D_{i}$, overlap with the sources for the number of trips per day, $T$. Those results are reported as individual bee averages and do not account for daily intra-individual variability.

The revised model input for the number of trips per day, $T$, has a lower average (mean of 2.91 compared with 10) and greater range than the assumption used by the Agencies (Figure 1A).

Perry et al. (2015) reported the average total number of trips recorded per bee (over her lifetime) between approximately 15 and 50. Lach et al. (2015) reported total trips up to 98 over a 4-week experiment, with medians of 12 and 13 trips per forager lifetime. By comparison, the Agencies assumed a mean of 10 trips per day. Averages of 5-10 flying days (Thompson et al. 2016) with approximately 3 trips per day equate to 15 to 30 trips per forager lifetime on average which fall within the range of reported values from Perry et al. (2015).

Based on quantitative data from six studies, a mean of $0.591 \pm 2.76 \mathrm{~h}$ per trip $\left(D_{i}\right)$ was calculated ( $n=3329$ for sample mean, $n=3320$ for sample standard deviation; Heberle 1914; Ribbands 1949; Eckert et al. 1994; Perry et al. 2016;
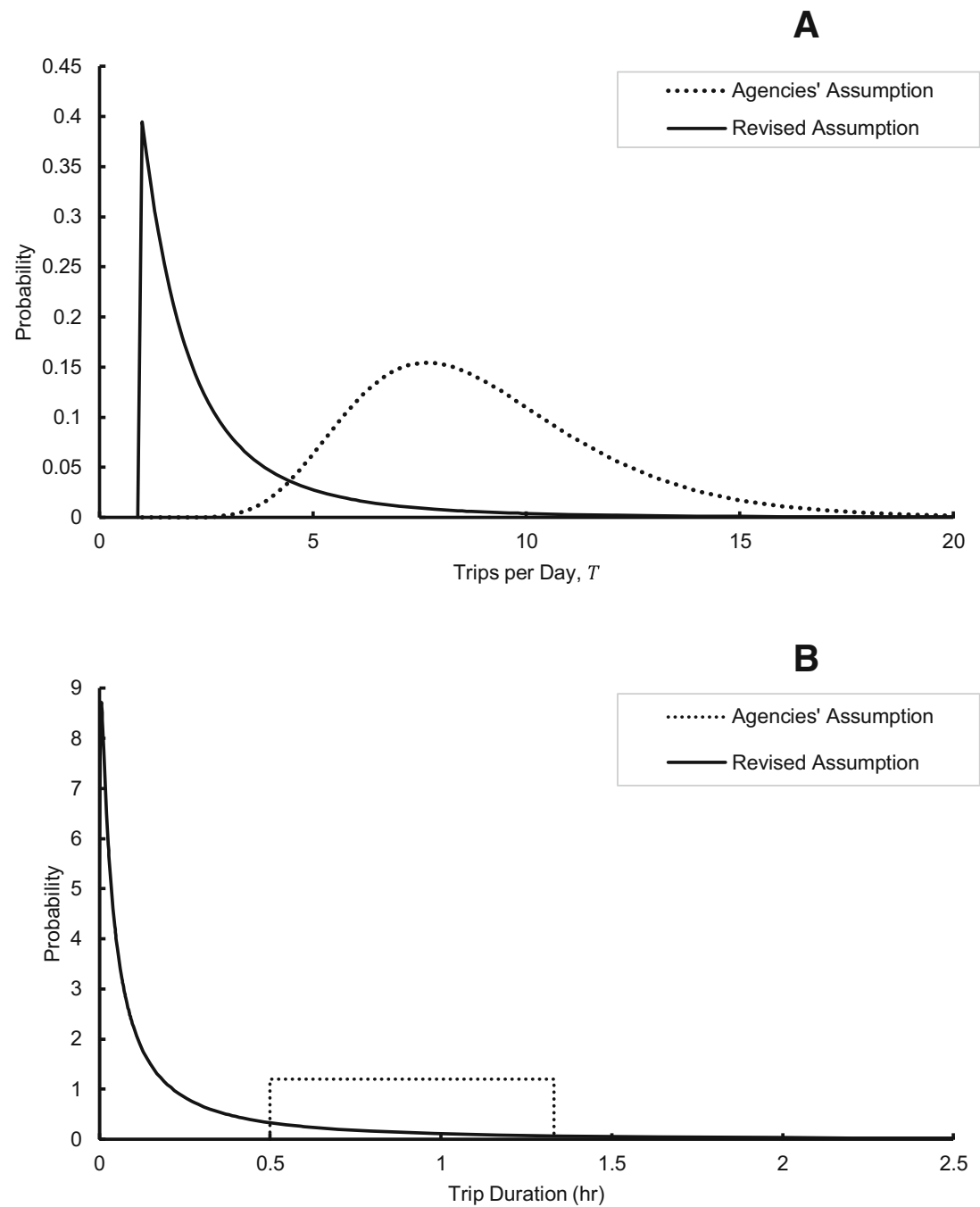

Figure 1. Probability density functions for trip model inputs: (A) trips per day, $T$, and (B) trip duration, $D_{i}$. 
Dosselli et al. 2016; Thompson et al. 2016). Of the other relevant studies, Park (1922) reported two mean trip durations of $0.567 \mathrm{~h}$ and $0.817 \mathrm{~h}$. Lundie (1925) estimated a range of 0.150 to $1.73 \mathrm{~h}$. The highest reported single trip duration observed was $2.5 \mathrm{~h}$ (Ribbands 1949). These results are in line with the data used to estimate $D_{i}$ (Online Resource 1, Table S1-2 Data used to estimate distribution parameters for average trip duration $\left(D_{i}\right)$ for honey bee nectar foragers in the RARI model). A lognormal distribution was assumed. For each trip, the distribution was truncated at $15 \mathrm{~h}$ (assumed daylight). Also, the number of trips was limited such that the sum of trip durations $\left(D_{i}\right)$ was not allowed to exceed $15 \mathrm{~h}$ (in this case, the maximum number of trips that did not exceed $15 \mathrm{~h}$ was taken in lieu).

In contrast to the Agencies' uniform distribution assumption, the revised model input assumption is right skewed with a much larger range (Figure 1B).

The fraction of trips spent flying, $F_{i}$, was not modified from the Agencies' assumption of a uniform distribution ranging from 0.5 to 0.9 . There are insufficient data to estimate this distribution, and the Agencies' assumption was based on a supposition of Rortais et al. (2005).

\subsubsection{Sugar requirements, $S_{F}$ and $S_{R}$}

For sugar requirements for flight, $S_{\mathrm{F}}$, we pooled sample means and variances from energetics studies that reported values for bees in free, untethered flight (Coelho and Mitton 1988; Wolf et al. 1989; Balderrama et al. 1992; Feuerbacher et al. 2003; Table I). Sample values originated from four studies reporting on 216 measurements taken from 125 bees (Online Resource 1, Table S1-3 Data used to estimate distribution parameters for sugar requirement for flight $\left(S_{\mathrm{F}}\right)$ of honey bee nectar foragers in the RARI model). We assumed a lognormal distribution, given the restriction to positive values and observed high variance, and estimated a mean of $12.6 \pm$ $6.10 \mathrm{mg}$ sugar/h.

The revised assumption for $S_{\mathrm{F}}$ has a mean that exceeds the Agencies' maximum value, a wider range, and a right skew compared with the
Agencies' uniform distribution assumption (Figure 2A).

For this model, it was not the intent to quantify true resting metabolism for $S_{\mathrm{R}}$. True resting metabolism is the energy consumption associated with motionless and not actively thermoregulating bees. True resting metabolism occurs when there are comparable thoracic and ambient air temperatures. In the open literature, there has been some confusion over resting metabolic rates because many authors have included motionless or resting bees that were also likely thermoregulating and therefore not at complete rest (see Rothe and Nachtigall 1989). We anticipate that nectar foragers will actively thermoregulate as needed when they are not foraging. In the absence of robust data for time spent thermoregulating, here we assume that any measures of relatively motionless bees reflect a random sample of non-flight metabolism of nectar foragers. As discussed in Rodney and Purdy (this issue), metabolism generally increases with increasing activity level. Nectar foragers that are not in flight may spend time at levels of energy expenditure greater than resting levels. However, in this simple model, a gradient of energy expenditure is purposely omitted.

From available studies meeting our criteria, a mean of $0.372 \pm 0.576 \mathrm{mg}$ sugar/h/bee was calculated from 329 measurements taken from 243 bees (Cahill and Lustik 1976; Rothe and Nachtigall 1989; Goller and Esch 1991; Stabentheiner et al. 2003; Kovac et al. 2007; Online Resource 1, Table S1-4 Data used to estimate the distribution parameters for sugar required to meet resting metabolic rate $\left(S_{\mathrm{R}}\right)$ of honey bee nectar foragers in the RARI and RP models). We believe that these estimates for resting metabolism overestimate energy expenditure of resting bees in the hive during the growing season because bees in clusters tend to have lower metabolism than isolated bees (Southwick 1983; Fahrenholz et al. 1989). Most bees from which resting metabolism was measured were isolated.

Southwick (1982) reported a range of 3.4 to $33.5 \mathrm{~W} / \mathrm{kg}$ of honey bees in the hive. This study included air temperatures ranging from -20 to over $25{ }^{\circ} \mathrm{C}$ and also measured energy consumption over full 24-h periods, with and without brood. The range reported translates to 

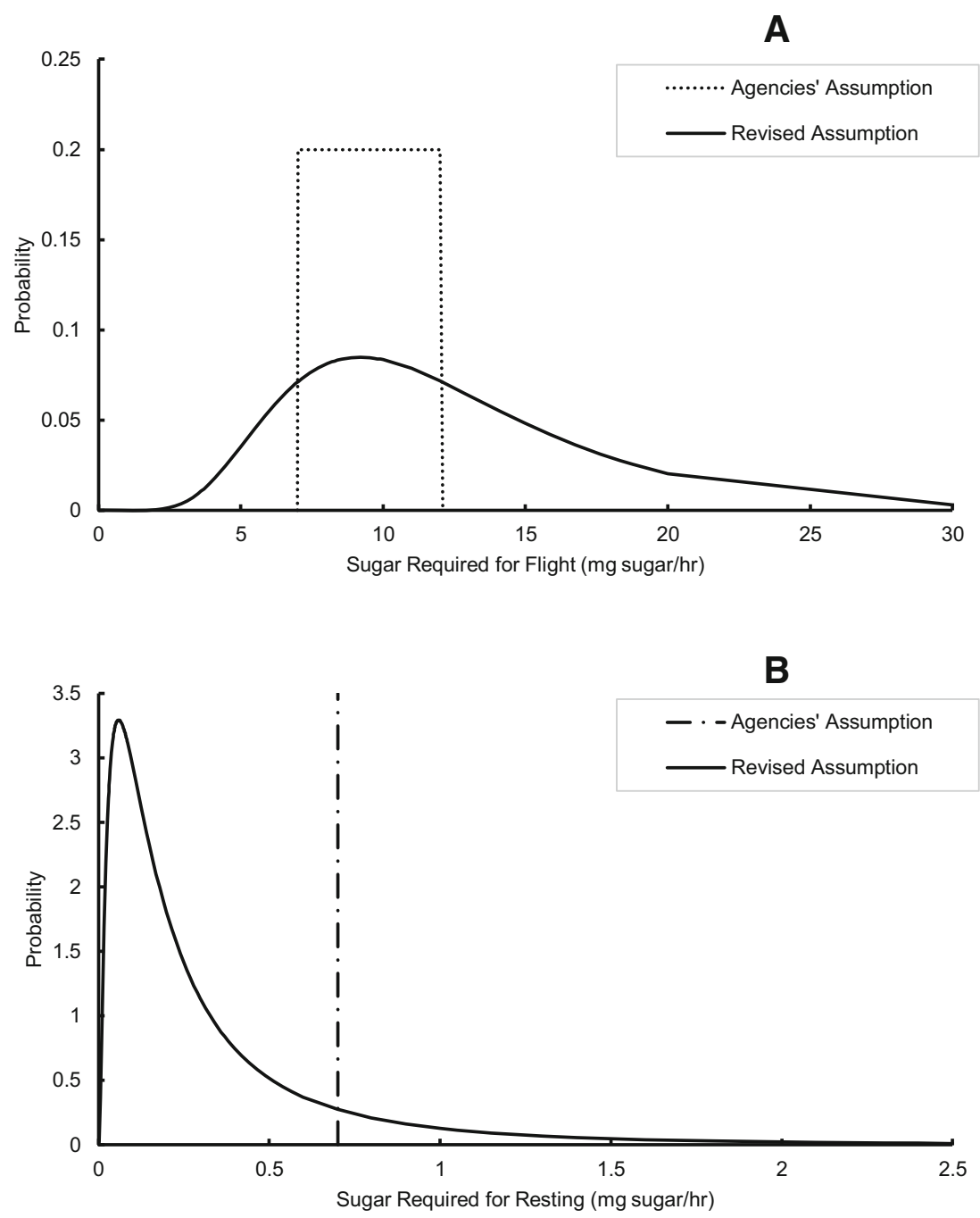

Figure 2. Probability density functions for sugar requirements: (A) for flight (mg/h), $S_{F}$, and (B) for resting (mg/h), $S_{\mathrm{R}}$.

approximately 0.072 to $0.72 \mathrm{mg}$ sugar/h per $100 \mathrm{mg}$ bees. This range is comparable to our estimates, despite the inclusion of air temperatures below those expected during the growing season in the USA. Given the relatively high observed variability and requirement for positive metabolism values, we assumed that the distribution for resting metabolism has a right skew. Thus, we assumed a lognormal distribution. The distribution assumption for sugar required to meet resting metabolic rate, $S_{\mathrm{R}}$, derived here for the RARI model was also subsequently used in the RP model as the base assumption for sugar required inside the hive, $S_{\mathrm{I}}$. For sugar requirements, upper and lower non-zero thresholds were not set for model inputs (Tables I and II) but were explored in subsequent sensitivity analyses.

Compared with the Agencies' assumption for resting metabolic rate, $S_{\mathrm{R}}$, of $0.7 \mathrm{mg}$ sugar/h, the revised assumption is probabilistic with a right skew and mean value lower than the Agencies' deterministic value (Figure 2B). 
Table II. Summary of input assumptions for the RP model for honey bee nectar forager nectar requirement estimates

\begin{tabular}{llllll}
\hline Input variable & Distribution & Min & Max & Mean & Standard deviation \\
\hline Time outside the hive $(\mathrm{h}), T_{\mathrm{O}}$ & Lognormal & NA & NA & 2.08 & 7.49 \\
Sugar required for time outside the hive $(\mathrm{mg} / \mathrm{h}), S_{\mathrm{O}}$ & Lognormal & NA & NA & 9.41 & 7.16 \\
Sugar required inside the hive $(\mathrm{mg} / \mathrm{h}), S_{\mathrm{I}}$ & Lognormal & NA & NA & 0.37 & 0.58 \\
Sugar content of nectar $(\mathrm{w} / \mathrm{w}), P$ & Beta & 0.02 & 0.79 & 0.30 & 0.10 \\
\hline
\end{tabular}

\subsubsection{Sugar content of nectar, $P_{i}$}

For the sugar content of collected nectar during trip $i, P_{i}$, we retained the Agencies' mean and standard deviation of 0.3 and 0.1 , respectively. These values were based on the Agencies' review of the sugar content of nectars presented in the White Paper (EPA, PMRA and CDPR 2012). These data are not necessarily representative of nectar that would be collected by nectar foragers in US agroecosystems because the plant species listed were largely non-crop plants, and nectar foragers are expected to prefer flowers with high sugar content (Knopper et al. 2016; Rodney and Purdy, this issue). This issue was explored in the sensitivity analysis.

In contrast to the Agencies' approach using a lognormal distribution with minimum and maximum of 0.1 and 0.6 , respectively, we applied a beta distribution to the $P_{i}$ assumption, which was permitted to range from 0.02 to 0.79 (see Fig. S11 in Online Resource 1). The lower limit here represents the lowest value from the Agencies' review, and the upper limit, the solubility of fructose in water at $20{ }^{\circ} \mathrm{C}$ (Chen and Chou 1993). Sugar content of nectar has been reported as high as 0.84 (see review by Knopper et al. 2016), and average crop load contents have been reported as high as approximately 0.65 . Therefore, a higher threshold than established by the Agencies' value of 0.6 is expected based on available nectars and sugar content preferences of foragers (see review by Rodney and Purdy, this issue). The average sugar content of nectar, $P_{\text {ave }}$, in accordance with the Agencies, was retained as a point estimate of 0.30 .

Differences between the Agencies' assumptions and our revised model inputs are presented in Table I.

\subsection{RP model}

The RP model is a simplified version of the Agencies' model. It is based solely on the estimated amount of time spent outside the hive, the metabolism that occurs inside and outside the hive, and the proportion of sugar $(\mathrm{w} / \mathrm{w})$ in nectar. By using time outside the hive per day instead of number of trips and trip durations, we bypass the need to quantify a possible negative correlation between trips per day and duration. We also account for trips taken outside the hive that contribute to metabolic requirements but are not necessarily associated with nectar foraging (e.g., defecation and orientation flights). Given that the fraction of trip spent flying cannot currently be accurately quantified, a fraction of time spent outside the hive in which the forager would not be flying or foraging (i.e., a time outside resting) was not included in the model. The RP model is given in Eq. 2.

$$
D_{\text {nectar }}=\frac{\left(S_{\mathrm{O}} * T_{\mathrm{O}}\right)+\left(S_{\mathrm{I}} *\left[24-T_{\mathrm{O}}\right]\right)}{P}
$$

where

$D_{\text {nectar }} \quad$ Nectar requirement (mg nectar/bee/day) $S_{O} \quad$ Sugar required outside the hive $(\mathrm{mg} / \mathrm{h})$

$S_{I} \quad$ Sugar required inside the hive $(\mathrm{mg} / \mathrm{h})$

$T_{\mathrm{O}} \quad$ Time spent outside the hive (h/day)

$P \quad$ Proportion of sugar in nectar $(\mathrm{w} / \mathrm{w})$

The distributions for sugar required inside the hive $(\mathrm{mg} / \mathrm{h}), S_{\mathrm{I}}$, and sugar content of nectar (w/w), $P$, were assumed to be the same as in the RARI model for $S_{\mathrm{R}}$ and $P_{i}$.

Data collated to estimate time outside the hive, $T_{\mathrm{O}}$, was primarily derived from literature sources 
that were also used in establishing distributions for the number of trips per day, $T$, and trip duration, $D_{i}$, in the RARI model. These studies focused on the bee as the experimental unit, and measurements collected over numerous days were averaged on a per bee basis. The result is that sample distributions represent bee averages and do not capture intra-individual temporal variation. He et al. (2013) demonstrated that as bees age, they tend to spend more time outside the hive.

Based on available data from two comprehensive studies carried out in Australia and the UK with 1615 bees, we calculated a mean of $2.08 \pm$ $7.49 \mathrm{~h}$ of average amount of time spent outside the hive per day, which was used to parameterize $T_{\mathrm{O}}$ (Online Resource 1, Table S1-5 Data used to estimate the distribution parameters for time spent outside the hive $\left(T_{\mathrm{O}}\right)$ for honey bee nectar foragers in the RP model and Fig. S1-2). These values are in good agreement with $\mathrm{He}$ et al. (2013) who reported a maximum time for a single bee from a cohort of 47 bees surviving to 36 days of $6.25 \mathrm{~h}$. Thom et al. (2000) reported that of 300 tagged bees, over $70 \%$ were out of the hive for less than $4.5 \mathrm{~h}$. Given the high variance and the lower limit of $5 \mathrm{~s}$ to $1 \mathrm{~min}$ based on the default value used in both studies, we presume a rightskewed distribution. We also assume an upper limit of $15 \mathrm{~h}$ based on our fixed assumption of a central location about June solstice (June 20, Kansas City). We assumed a lognormal distribution (Online Resource 1, Fig. S1-6) and explored the impact of changing the distribution assumption and adding lower thresholds in the sensitivity analysis.

Thompson et al. (2016) reported that bees on average flew on 5.0 to 10.3 days only (observation period was 5 weeks). In total, bees were outside the hive on average 9.05 to $24.51 \mathrm{~h}$ across the entire observation period of 5 weeks (Thompson et al. 2016). These results are in line with Perry et al. (2015) who reported mean total hours outside the hive between 5 and $15 \mathrm{~h}$ (40-day observation period). Total lifetime spent outside the hive was up to $88 \mathrm{~h}$, with medians of 11 and $13 \mathrm{~h}$ reported by Lach et al. (2015). The lowest median age of the first forage in the study was 9 days, and the median age of the last forage was 23 days. Accordingly, it is reasonable to assume that foragers will forage over approximately 2 weeks, and of this time, one to two-thirds of days will typically involve flights outside the hive (for foraging and other activities). This is fairly consistent with the individual data presented by He et al. (2013; Figure 8b therein). Our assumption based on the available data was $\sim 2 \mathrm{~h}$ outside the hive per day (RP model), which is consistent with these data, in that $2 \mathrm{~h}$ per 5 to 10 flying days is equivalent to 10 to 20 total hours of time outside the hive. This range falls within the mean range of Thompson et al. (2016) and overlaps the high end of the range reported by Perry et al. (2015). By comparison, the RA model predicted a median of $8.71 \mathrm{~h} /$ day outside the hive. If we again assume an average of 5 to 10 flying days per forager, this would equate to 44 to $88 \mathrm{~h}$ outside the hive over the forager's lifetime. These estimates based on the Agencies' model exceed the upper bounds reported by Thompson et al. (2016), Perry et al. (2015), and Lach et al. (2015). The study by Lach et al. (2015) included monitoring of 447 bees.

An upper threshold of $15 \mathrm{~h} /$ day was specified for time outside the hive $\left(T_{\mathrm{O}}\right)$ and trip duration $\left(D_{i}\right)$ for both RP and RARI models. The variability in the underlying data was high due to some individual bees spending $>24$ h continuously outside the hive (Thompson, 2018; personal communication). In these cases, which were rare, it is unlikely that the bees were foraging for that long, and it is more likely that they drifted to a different hive or found cover overnight elsewhere. Ultimately, the upper threshold of $15 \mathrm{~h} /$ day for trip duration $\left(D_{i}\right)$ or time outside the hive $\left(T_{\mathrm{O}}\right)$ led to a lower mean and standard deviation $(1.68 \pm$ $2.94 \mathrm{~h}$ ) in the values used than originally estimated based on author reported values (2.07 \pm $7.49 \mathrm{~h}$ ).

The data used to parameterize the sugar required for flight, $S_{\mathrm{F}}$, distribution in the Agencies' model with revised inputs were used to estimate the sugar requirement outside the hive. In addition, data for bees foraging inside a large $(6 \mathrm{~L})$ respirometer (Balderrama et al. 1992) and flying between artificial flowers were also included as representative of outdoor behavior of foragers. A mean of $9.41 \pm 7.16 \mathrm{mg}$ sugar/h was calculated ( $n=769$; Online Resource 1, Table S1-6 Data used to estimate the distribution parameters for 
sugar required outside the hive $\left(S_{\mathrm{O}}\right)$ by honey bee nectar foragers for the RP model and Fig. S1-3).

Several energetics studies identified in Rodney and Purdy (this issue) were reviewed subsequent to the modeling effort presented herein. Most of the additional studies provided data that are consistent with those used in the modeling (see Online Resource 3 for Rodney and Purdy, this issue). However, most did not meet the criteria for inclusion in the quantitative modeling. Those that did (Heusner and Stussi 1964; Moffatt 2000) fell within the range of values used to estimated $S_{\mathrm{R}}$, $S_{\mathrm{F}}$, and $S_{\mathrm{O}}$. Their addition to the datasets used to estimated distributions would have negligible impact on model inputs and predictions and was omitted from the parameter distribution estimations.

Monte Carlo simulations were carried out with 100,000 trials per model.

\subsection{Exploratory sensitivity and correlation analyses}

When models contain fixed assumptions, sensitivity to variability in those assumptions can be explored by changing the inputs and observing how the results affect model predictions (Vose 1996). Alternatively, simulations can be generated that account for the potential distribution of inputs (e.g., Monte Carlo analyses, see EPA ( 2009)). In the case of the Agencies, the RA, RARI, and Rodney-Purdy (RP) nectar forager models, inputs are predominantly probabilistic and already account for estimated variability. A concern, given limited data, is how alternate assumptions about the distribution of a variable may impact (1) the model predictions, (2) the contribution to variance of the inputs, and (3) the correlation between inputs and predictions (Paleari and Confalonieri, 2016).

We investigated the effects on predictions when changing the mean or standard deviation of each input variable by $\pm 50 \%$, while holding other inputs constant. This type of sensitivity analysis, also known as an elasticity analysis, is used to determine those input variables for which consistent changes to the parameters have the most influence on model predictions. Depending on the input variable, we also explored changes to thresholds (minima and maxima), as well as different distribution assumptions (Online Resource 1, Table S17 Summary of exploratory sensitivity analyses of the RARI model with fixed changes to model assumptions and Table S1-8 Summary of exploratory sensitivity analyses of the Rodney-Purdy model with fixed changes to model assumptions). We consider here (1) impacts on model predictions, (2) contribution to variance, and (3) rank correlation.

Applying upper and lower thresholds to available data in the modeling can be problematic. For example, take specifying a lower threshold of $30 \mathrm{~min}$ for trip duration (or time outside the hive). The data used to estimate the distributions for trip duration and time outside the hive did not contain this threshold. Accordingly, means and standard deviations were based in part on trips and times outside the hive that were lower than this threshold. When this threshold is applied to the distribution defined by these data (for a lognormal distribution), the applied mean and standard deviation are retained, and the distribution is reshaped to meet all parameters. Clearly, this is not the same as removing trips or durations less than $30 \mathrm{~min}$ from the initial dataset. The lower limit of $30 \mathrm{~min}$ was selected for exploration in accordance with the Agencies' model; however, available data indicate that workers of foraging age will take trips outside the hive with durations far less than $30 \mathrm{~min}$.

Exploratory correlation analyses were conducted with the RA and RARI models. The correlations that were intuitively expected and explored included (1) high and moderate negative correlation between trips per day and trip duration (i.e., fewer trips are taken per day when trips are of longer duration), (2) high and moderate negative correlation between trip duration and fraction of trip spent flying (i.e., the proportion of a trip spent flying decreases with increasing trip duration), and (3) high forage fidelity (i.e., bees will forage on the same patch over multiple trips, and there is thus dependency among the number of trips on a given day). We assumed that trips on a given day would be the same length, with the same fraction of time spent flying and the same sugar content of nectar. The scenario in which all correlations listed above are concurrently high was also run. 
High correlation coefficients were set at -1.0 , and moderate correlation coefficients were set at -0.5 .

\section{RESULTS}

\subsection{Agencies' model}

We were unable to exactly reproduce the percentile estimates generated by the Agencies for nectar forager daily nectar requirements (Online Resource 2, Table S2-1 Nectar requirement estimates for nectar foragers $\left(D_{\text {nectar }}\right)$ with the RA model). We estimated a median of $253 \mathrm{mg}$ nectar/ bee/day, which is $13 \%$ lower than the Agencies' value of $292 \mathrm{mg}$ nectar/bee/day, and a mean of $265 \pm 78.1 \mathrm{mg}$ nectar/bee/day. In general, our percentile estimates were between 6 and 19\% lower than those reported in the Agencies' White Paper (EPA, PMRA and CDPR 2012), with difference generally increasing with increasing percentiles of the distributions. Note the Agencies did not report means or standard deviations for comparison. With our reproduction of the Agencies' model, we estimated a mean total flying time of $6.39 \pm$ $2.03 \mathrm{~h}$ and a cumulative total daily trip time of $9.13 \pm 2.86 \mathrm{~h}$. The Agencies reported a median flying time of $8.9 \mathrm{~h}$; by comparison, we estimated a median flying time of $6.10 \mathrm{~h}$ with our reproduction of their model.

Assuming a negative correlation between trips per day, $T$, and duration, $D_{i}$, resulted in notably lower variability in predictions of nectar requirement but had little impact on predicted medians and means $(<6 \%$ lower). Assuming a negative correlation between trip duration, $D_{i}$, and fraction of trip spent flying, $F_{i}$, also resulted in lower variability and lower central values, though even less pronounced. All tabled percentile estimates were slightly lower than the base case (Online Resource 2, Table S2-23 Nectar requirement estimates for nectar foragers $\left(D_{\text {nectar }}\right)$ from the RA model with explored correlations and Figs. S2-16 and S2-17).

The fidelity assumption removed variability between numbers of trips in a day and assumed all trips would be of the same duration, $D$, the same fraction of trip spent flying, $F$, and the same sugar content of nectar, $P$. This assumption resulted in much lower predictions of nectar requirement up to approximately the 65 th percentile but at and beyond the 75th percentile resulted in higher predictions (Online Resource 2, Table S223 Nectar requirement estimates for nectar foragers $\left(D_{\text {nectar }}\right)$ from the RA model with explored correlations and Fig. S2-18). Overall the impact was greater variability with the same predicted mean and a predicted median that was approximately $6 \%$ lower than the base case.

Assuming high fidelity and high negative correlations between trip duration, $D_{i}$, and frequency, $T$, and fraction of trip spent flying, $F_{i}$, resulted in slightly lower variability in predictions than base case and also lower estimates of nectar requirement. The mean declined from the base case of 265 to $250 \mathrm{mg}$ nectar/bee/day, and the median declined from 253 to $239 \mathrm{mg}$ nectar/bee/day (Online Resource 2, Table S2-23 Nectar requirement estimates for nectar foragers ( $\left.D_{\text {nectar }}\right)$ from the RA model with explored correlations and Fig. S2-19).

\subsection{Agencies' model with revised inputs}

The RARI model resulted in a much larger range of predicted values, with a considerable right skew (Figure 3A, B). The median estimate from the RARI model is $43.6 \mathrm{mg}$ nectar/bee/day, over $6 \times$ lower than the Agencies' estimate of $292 \mathrm{mg}$ nectar/bee/day. From the RARI model, the mean nectar requirement was $75.2 \pm 100 \mathrm{mg}$ nectar/bee/day. The assumption with the largest contribution to variance in predicted nectar requirement with the RARI model is the sugar required to meet resting metabolic rate, $S_{\mathrm{R}}$, with a value of $44 \%$. The next largest contributor to variance is trip duration $\left(D_{i}\right)$, with a value of $26 \%$, followed by the number of trips per day (T), 20\%; the amount of sugar required for flight $\left(S_{\mathrm{F}}\right), 6.2 \%$; the sugar content of nectar $\left(P_{i}\right)$, $2.5 \%$; and the proportion of the trip spent flying $\left(F_{i}\right), 1.3 \%$. Ranked correlation follows this same pattern in terms of magnitude, except, as expected, the sugar content of nectar collected during trips $\left(P_{i}\right)$ is negatively correlated with the predicted nectar requirement (Online Resource 2, Table S2-3 Sensitivity of nectar requirement estimates $\left(D_{\text {nectar }}\right)$ to the RARI model inputs). 

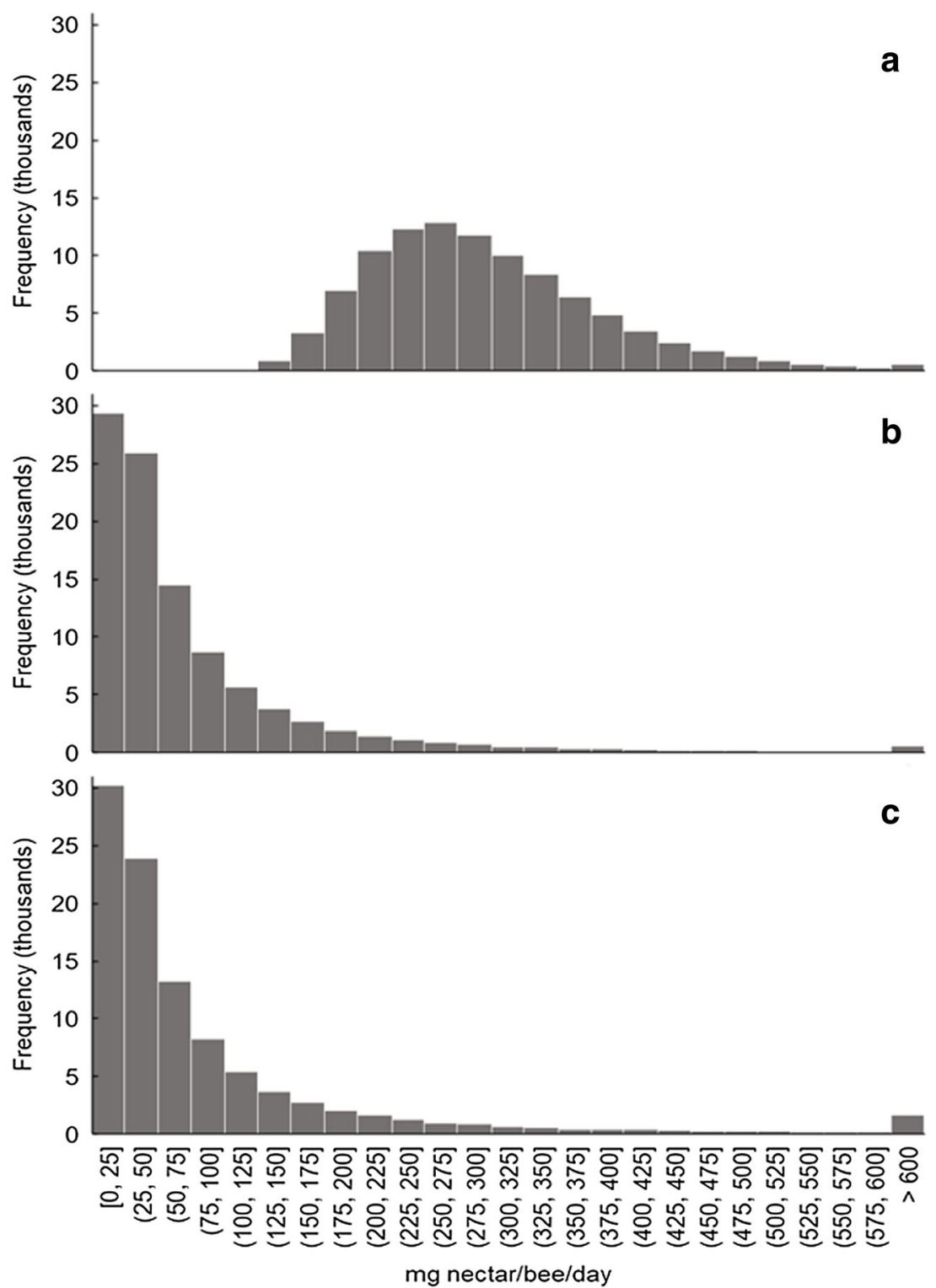

Figure 3. Frequency distributions for model results of (A) the RA model, (B) the RARI model, and (C) the RP model.

\subsubsection{Number of trips per day, $T$}

For the number of trips per day $(T)$ in the RARI model, most changes to the input distribution (i.e., switch to beta distribution, lower maximum number of trips per day, changing the variance) had a negligible impact on model predictions (Online Resource 2, Table S2-5 Nectar requirement estimates for nectar foragers $\left(D_{\text {nectar }}\right)$ from the RARI model with explored assumptions for the number of trips per day $(T)$ (mg nectar/bee/day) and Figs. S2-2 through S2-4). Changing the mean by $\pm 50 \%$, however, resulted in a considerable difference with mean and median predictions for nectar requirement of 51.6 to $98.8 \mathrm{mg}$ nectar/bee/ day and 28.8 to $60.0 \mathrm{mg}$ nectar/bee/day, respectively (Online Resource 2, Fig. S2-2). From the sensitivity analyses, the ordering of contribution to variance and rank correlation did not change for any of the explored changes, except for when the 
high mean was applied. This resulted in trip duration $\left(D_{i}\right)$ having the highest contribution to variance and the highest rank correlation (as opposed to sugar required to meet resting metabolic rate $\left(S_{\mathrm{R}}\right)$ ) (Online Resource 2, Table S2-6 Contribution to variance for RARI model inputs with explored assumptions for the number of trips per day $(T)$ and Table S2-7 Rank correlation for RARI model inputs with explored assumptions for the number of trips per day $(T))$.

\subsubsection{Sugar required for flight, $S_{F}$}

The only change to the distribution for sugar required for flight $\left(S_{\mathrm{F}}\right)$ that substantially affected model predictions was changing the mean by \pm 50\% (Online Resource 2, Table S2-8 Nectar requirement estimates for nectar foragers $\left(D_{\text {nectar }}\right)$ from the RARI model with explored assumptions for sugar required for flight $\left(S_{\mathrm{F}}\right)$ and Figs. S2-5 to $\mathrm{S} 2-7)$. The mean and median predictions from changing the mean $\pm 50 \%$ were 51.4 to $98.8 \mathrm{mg}$ sugar/bee/day and 30.3 to $54.9 \mathrm{mg}$ sugar/bee/day, respectively.

Only the high mean changed the ordering of contributions to variance and rank correlation. Increasing the mean by $50 \%$ resulted in trip duration having the highest contribution to variance and the highest correlation with nectar requirement.

\subsubsection{Sugar required to meet resting metabolic rate, $S_{R}$}

The lower percentile predictions of nectar requirement were particularly sensitive to changes in the distribution parameters for sugar required to meet resting metabolic rate, $S_{\mathrm{R}}$ (Online Resource 2, Table S2-11 Nectar requirement estimates for nectar foragers $\left(D_{\text {nectar}}\right)$ from the RARI model with explored assumptions for sugar required for rest $\left(S_{\mathrm{R}}\right)$ and Figs. S2-8 through S2-10). High mean, low standard deviation, and setting a minimum threshold of $0.1 \mathrm{mg}$ sugar/h led to higher predictions for nectar requirements at lower percentiles, and in the case of the high mean, also higher percentiles. The Weibull model assumption, high standard deviation, and low mean led to lower predictions of nectar requirement at lower percentiles and also at higher percentiles in the case of the low mean assumption. Changing the mean $\pm 50 \%$ resulted in the widest range of predicted measures of centrality, i.e., means and medians of 61.6 to $89.5 \mathrm{mg}$ nectar/bee/day and 28.5 to $60.1 \mathrm{mg}$ nectar/bee/day, respectively.

Sugar required to meet resting metabolic rate $\left(S_{\mathrm{R}}\right)$ had the highest contribution to variance and the highest rank correlation of all input variables in the base case and also across all other changes to the input distribution, except when reducing the mean by $50 \%$. The latter scenario resulted in trip duration, $D_{i}$, contributing more to variance and having the highest rank correlation among the input variables (Online Resource 2, Table S2-12 Contribution to variance for RARI model inputs with explored assumptions for the sugar required for rest $\left(S_{\mathrm{R}}\right)$ and Table S2-13 Rank correlation for RARI model inputs with explored assumptions for the sugar required for rest $\left(S_{\mathrm{R}}\right)$ ).

\subsubsection{Duration of trips, $D_{i}$}

Changing the lower threshold for trip duration $\left(D_{i}\right)$ to $1 \mathrm{~min}$ had a negligible effect on the predictions for nectar requirement. However, increasing the minimum threshold to $30 \mathrm{~min}$ had a larger effect on predictions, particularly the lower percentiles (Figure 4). Assuming a Weibull model generally decreased predictions of nectar requirement in the central percentiles. Changes $( \pm 50 \%)$ to the mean or standard deviation had a clear impact on nectar requirement predictions, with changes to the mean resulting in greater effect (Online Resource 2, Table S2-14 Nectar requirement estimates for nectar foragers $\left(D_{\text {nectar }}\right)$ from the RARI model with explored assumptions for trip duration $\left(D_{i}\right)$ and Figs. S2-11 and S2-12). Of all the elasticity analyses, the greatest mean nectar requirement stemmed from the high mean scenario $(98.8 \mathrm{mg} \mathrm{nectar} / \mathrm{bee} / \mathrm{h})$, and the highest median arose with the assumption of a minimum of $30 \mathrm{~min}$ per trip (61.6 mg nectar/bee/h).

For each of the changes to the input distribution for trip duration $\left(D_{i}\right)$, the order of contribution to variance and rank correlations did not change from the base case, except for the high mean scenario. The high mean scenario resulted in trip duration $\left(D_{i}\right)$ contributing the 


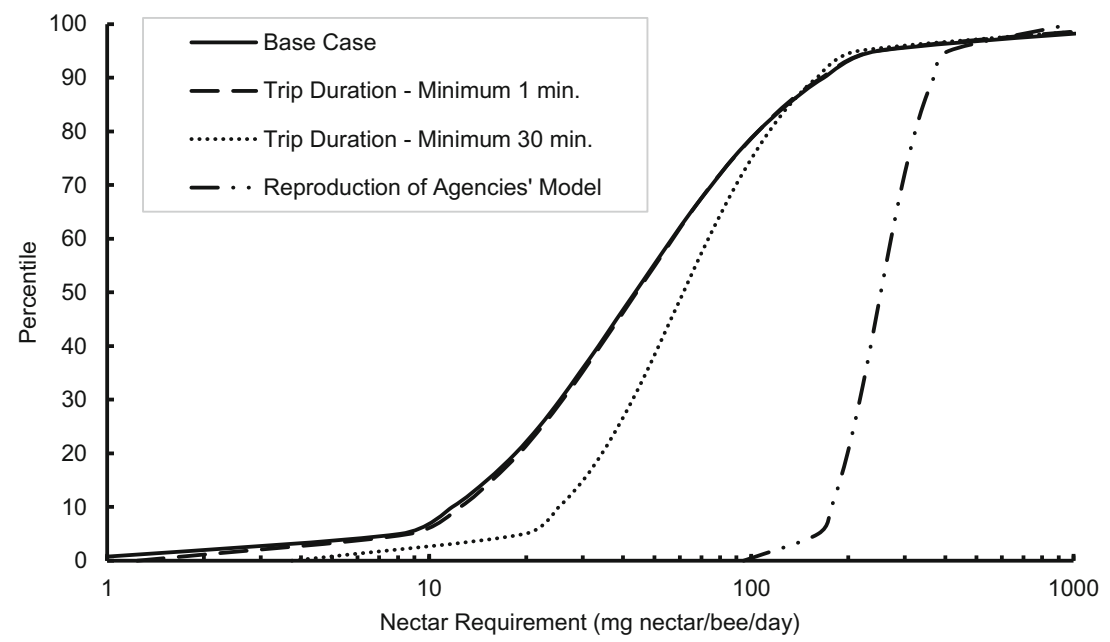

Figure 4. Predicted cumulative distributions of nectar requirement for nectar-foraging honey bees with changes to the lower threshold assumption for trip duration $\left(D_{i}\right)$ in the RARI model.

most to the variance in nectar requirement and also resulted in trip duration $\left(D_{i}\right)$ having the highest rank correlation with nectar requirement (Online Resource 2, Table S2-15 Contribution to variance for RARI model inputs with explored assumptions for trip duration $\left(D_{i}\right)$ and Table S2-16 Rank correlation for RARI model inputs with explored assumptions for trip duration $\left(D_{i}\right)$ ).

\subsubsection{Fraction of trip spent flying, $F_{i}$}

The assumed changes to the distribution for fraction of trip spent flying had little impact on the predicted nectar requirements (Online Resource 2, Table S2-17 Nectar requirement estimates for nectar foragers $\left(D_{\text {nectar }}\right)$ from the RARI model with explored assumptions for fraction of trip spent flying $\left(F_{i}\right)$ and Fig. S2-13). The ranges of means and medians were 65.2 to $85.2 \mathrm{mg}$ nectar/bee/day and 39.3 to $47.8 \mathrm{mg}$ nectar/bee/day, respectively. The changes to the input variable for fraction of trip spent flying did not alter the ordering of contributions to variance or rank correlations of input variables (Online Resource 2, Table S2-18 Contribution to variance for RARI model inputs with explored assumptions for fraction of trip spent flying $\left(F_{i}\right)$ and Table S2-19
Rank correlation for RARI model inputs with explored assumptions for fraction of trip spent flying $\left(F_{i}\right)$ ).

\subsubsection{Sugar content of nectar, $P_{i}$ and $P_{\text {ave }}$}

Changes of $\pm 50 \%$ to the standard deviation for sugar in nectar did not dramatically change the predictions for nectar requirement; however, changing the mean $\pm 50 \%$ did result in substantial changes to the prediction of nectar requirement throughout most of the distribution. Mean and median ranges were 60.3 to $321 \mathrm{mg}$ nectar/bee/ day and 28.3 to $98.4 \mathrm{mg}$ nectar/bee/day, respectively (Online Resource 2, Table S2-20 Nectar requirement estimates for nectar foragers $\left(D_{\text {nectar }}\right)$ from the RARI model with explored assumptions for sugar content of nectar $\left(P_{i}\right)$ and Figs. S2-14 and S2-15).

No changes to the order of contribution to variance or rank correlation of the model input variables were observed with changes to the input distribution for sugar content of nectar (Online Resource 2, Table S2-21 Contribution to variance for RARI model inputs with explored assumptions for sugar content of nectar $\left(P_{i}\right)$ and Table S2-22 Rank correlation for RARI model inputs with explored assumptions for sugar content of nectar $\left.\left(P_{i}\right)\right)$. 


\subsubsection{Correlation analyses}

Assuming a negative correlation between trips per day, $T$, and duration, $D_{i}$, resulted in notably lower variability in predictions of nectar requirement and generally lower predicted nectar requirements, in contrast to the results for the RA model. Assuming a negative correlation between trip duration and fraction of trip spent flying did not result in much change to predictions of nectar requirement, which was similar for the RA model. The fidelity assumption resulted in lower predictions of nectar requirement throughout the distribution (Online Resource 2, Table S2-24 Nectar requirement estimates for nectar foragers $\left(D_{\text {nectar }}\right)$ from the RARI model with explored correlations and Figs. S2-20 through S2-21).

Assuming high negative correlations between trip duration, $D_{i}$ and frequency, $T$, and fraction of trip spent flying, $F_{i}$, resulted in generally lower estimates of nectar requirement. The mean declined from the base case of 75.2 to $46.3 \mathrm{mg}$ nectar/bee/day, and the median declined from 43.6 to $29.8 \mathrm{mg}$ nectar/bee/day (Online Resource 2, Table S2-24 Nectar requirement estimates for nectar foragers $\left(D_{\text {nectar }}\right)$ from the RARI model with explored correlations and Figs. S2-20 through S2-22).

\subsection{RP model}

The RP model predictions for nectar requirements of nectar foragers are similar to that of the RARI model (Figure 3B and C; Online Resource 3, Table S3-1 Nectar requirement estimates for nectar foragers $\left(D_{\text {nectar }}\right)$ with the Rodney-Purdy model). The median estimate from the RP model is $44.4 \mathrm{mg}$ nectar/bee/day. From the RP model, the mean nectar requirement was $92.4 \pm 161 \mathrm{mg}$ nectar/bee/day, and the mean time outside the hive was $1.68 \pm 2.94 \mathrm{~h}$. The assumption with the largest contribution to variance in predicted nectar requirement with the RP model is the time outside the hive, $T_{\mathrm{O}}$, with a value of 0.49 (Online Resource 3, Table S3-2 Sensitivity of nectar requirement estimates for nectar foragers $\left(D_{\text {nectar }}\right)$ to the Rodney and Purdy model inputs). The next largest contributor to variance is sugar required inside the hive $\left(S_{\mathrm{I}}\right)$, with a value of 0.32 , followed by the sugar content of nectar $(P), 0.11$, and the sugar required outside the hive $\left(S_{\mathrm{O}}\right), 0.087$. Ranked correlation follows this same pattern in terms of magnitude.

\subsubsection{Sugar required outside the hive, $S_{o}$}

The only input modification that substantially affected predicted nectar requirement was changing the input mean $\pm 50 \%$ (Online Resource 3, Table S3-3 Nectar requirement estimates for nectar foragers $\left(D_{\text {nectar }}\right)$ from the Rodney-Purdy model with explored assumptions for sugar required outside the hive $\left(S_{\mathrm{O}}\right)$ and Figs. S3-1 through S3-3). The resulting mean range was 62.4 to $107 \mathrm{mg}$ nectar/bee/day, and the median range was 30.6 to $61.7 \mathrm{mg}$ nectar/bee/day.

The order of contribution to variance and rank correlation did not change from the base case for any changes to the input variable, except for the low mean scenario (Online Resource 3, Table S34 Contribution to variance for Rodney-Purdy model inputs with explored assumptions for sugar required outside the hive $\left(S_{\mathrm{O}}\right)$ and Table S3-5 Rank correlation for Rodney-Purdy model inputs with explored assumptions for sugar required outside the hive $\left.\left(S_{\mathrm{O}}\right)\right)$. A low mean for sugar required outside the hive resulted in sugar required inside the hive having the highest contribution to variance and the highest rank correlation with the predicted nectar requirement (over time outside the hive).

\subsubsection{Sugar required inside the hive, $S_{I}$}

Modifying the distribution for sugar inside the hive had considerable impact on the predictions of nectar requirement (Online Resource 3, Nectar requirement estimates for nectar foragers $\left(D_{\text {nectar }}\right)$ from the RodneyPurdy model with explored assumptions for sugar required inside the hive $\left(S_{\mathrm{I}}\right)$ and Figs. S3-4 through S3-6). This was particularly true for adjustments not only to the mean but also to the lower percentiles of nectar requirement with changes to the standard deviation, change to Weibull distribution, and implementation of a lower threshold of $0.1 \mathrm{mg}$ sugar/h. When the 
input mean was altered $\pm 50 \%$, the mean nectar requirement ranged from 75.9 to $107 \mathrm{mg} \mathrm{nec-}$ tar/bee/day, and the corresponding median ranged from 28.0 to $61.7 \mathrm{mg}$ nectar/bee/day.

None of the modifications to the distribution for sugar required inside the hive impacted the ordering of contributions to variance or rank correlation of input variables (Online Resource 3, Table S3-7 Contribution to variance for RodneyPurdy model inputs with explored assumptions for sugar required inside the hive $\left(S_{\mathrm{I}}\right)$ and Table S3-8 Rank correlation for Rodney-Purdy model inputs with explored assumptions for the sugar required inside the hive $\left(S_{\mathrm{I}}\right)$ ).

\subsubsection{Proportion of sugar in nectar, $P$}

Changes to the standard deviation assumption for sugar content of nectar, $P$, had little impact on predictions of nectar requirement. However, changing the mean to $15 \%$ or to $45 \%$ from the base assumption of $30 \%$ had a considerable effect on predicted nectar requirements (Figure 5; Online Resource 3, Table S3-9 Nectar requirement estimates for nectar foragers $\left(D_{\text {nectar }}\right)$ from the Rodney-Purdy model with explored assumptions for the sugar content of nectar $(P)$ and Figs. S3-7 and S3-8). In the latter case, mean range was 56.8 to $265 \mathrm{mg}$ nectar/bee/day, and the median range was 28.4 to $105 \mathrm{mg}$ nectar/bee/day.
No changes to the distribution for sugar in nectar, $P$, affected the order from base case for contributions to variance or rank correlation of input variables (Online Resource 3, Table S3-10 Contribution to variance for Rodney-Purdy model inputs with explored assumptions for the sugar content of nectar $(P)$ and Table S3-11 Rank correlation for Rodney-Purdy model inputs with explored assumptions for the sugar content of nectar $(P))$.

\subsubsection{Time outside the hive, $T_{o}$}

Setting a lower threshold of 1 min for time outside the hive $\left(T_{\mathrm{O}}\right)$ had a negligible impact on the predictions for nectar requirement. However, setting the lower threshold to $30 \mathrm{~min}$ decreased the variability and generally increased the predictions for nectar requirement. Assuming a Weibull distribution not only led to generally decreased predictions through the center of the distribution but also increased variability in nectar requirement. Changes to the mean and standard deviation led to notable changes in predicted nectar requirement. Modifying the mean $\pm 50 \%$ had the most significant impact with a resulting mean range in nectar requirement of 61.4 to $124 \mathrm{mg}$ nectar/bee/ day and a median range of 29.3 to $62.7 \mathrm{mg}$ nectar/ bee/day (Online Resource 3, Table S3-12 Nectar requirement estimates for nectar foragers ( $\left.D_{\text {nectar }}\right)$

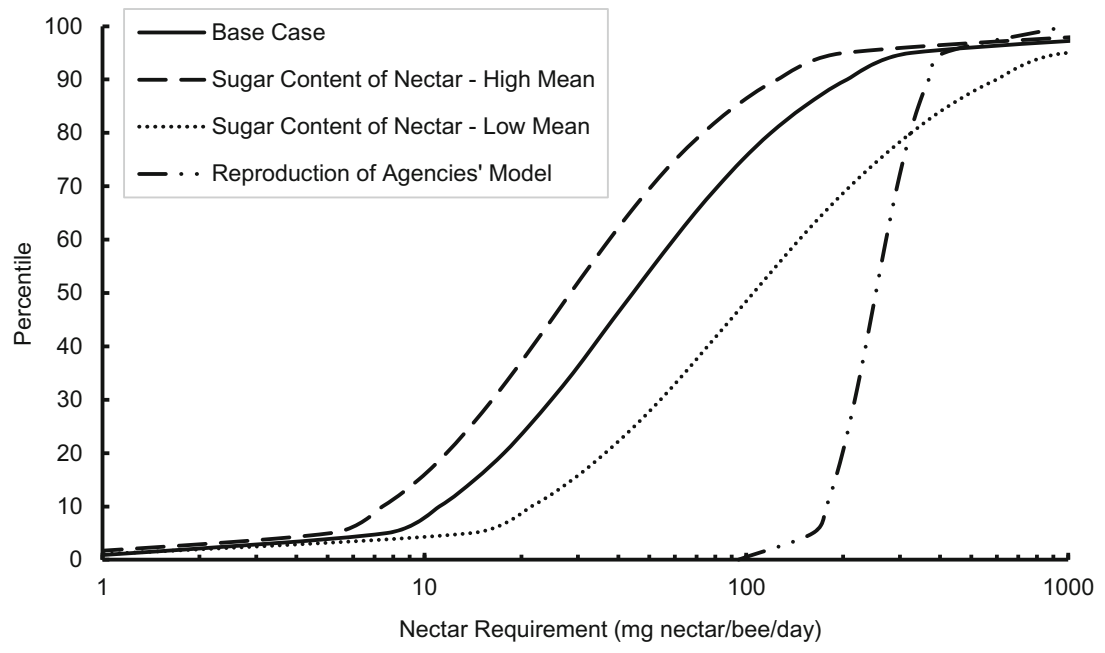

Figure 5. Predicted cumulative distributions of nectar requirement for nectar-foraging honey bees with changes to the mean assumption for sugar content of nectar $(P)$ in the RP model. 
from the Rodney-Purdy model with explored assumptions for the time spent outside the hive $\left(T_{\mathrm{O}}\right)$ and Figs. S3-9 through S3-11).

For the most part, there were no changes to the order of contribution to variance or rank correlation of input variables across this exploration of time outside the hive $\left(T_{\mathrm{O}}\right)$. However, both the low mean and minimum threshold of $30 \mathrm{~min}$ led to sugar requirement overtaking time outside the hive for both contribution to variance and rank correlation (Online Resource 3, Table S3-13 Contribution to variance for Rodney-Purdy model inputs with explored assumptions for the time spent outside the hive $\left(T_{\mathrm{O}}\right)$ and Table S3-14 Rank correlation for Rodney-Purdy model inputs with explored assumptions for the time spent outside the hive $\left.\left(T_{\mathrm{O}}\right)\right)$.

\subsubsection{Multiple changes to RP input distributions}

We explored the impact of setting all mean inputs to conservatively high values $(+50 \%$ of estimated mean, $-50 \%$ for sugar content). This resulted in a major increase from the base case for predicted nectar requirements, to the point of intersection with the Agencies' predictions but with greater variability (Online Resource 3, Table S315 Nectar requirement estimates for nectar foragers $\left(D_{\text {nectar }}\right)$ from the Rodney-Purdy model with explored changes to multiple assumptions and Fig. S3-12). This scenario did not result in any changes to the order of contribution to variance or rank correlation (Online Resource 3, Table S3-16 Contribution to variance for RodneyPurdy model inputs with explored changes to multiple assumptions and Table S3-17 Rank correlation for Rodney-Purdy model inputs with explored changes to multiple assumptions).

Subsequently, we changed the above scenario to also include a lower threshold of $30 \mathrm{~min}$ for time outside the hive, $T_{\mathrm{O}}$. This modification resulted in further increases to predictions of nectar requirement, particularly in the lower portion of the distribution. This scenario also resulted in the sugar content of nectar taking top rank in contribution to variance and rank correlation with predictions.

\section{DISCUSSION}

That the RARI model estimates are similar to those of the RP model suggests that the differences between the RP model and the Agencies' model are predominantly due to model parameterization, as opposed to model form. That said, the Agencies' model specifies seven different model parameters, three of which are varied within individual (bee) simulations $\left(D_{i}, F_{i}\right.$, and $\left.P_{i}\right)$. The RP model achieves similar predictions with a simpler model, with only five variables, suggesting that the Agencies' model is overly complex for the available data. Further, the fraction of trip spent flying, $F_{i}$, a parameter found in the Agencies' model, is a supposition that is not documented to be based on any real-world data. The proportion of sugar content of nectar during trip $i, P_{i}$, is also a problematic variable, given the evidence that foragers take concentrated nectar from the hive to fuel part or all of their foraging trips (Harano and Nakamura 2016). In part, the similarity between the predictions of the RARI and RP models can be attributed to the fact that many of the data used to parameterize the models were acquired from the same studies.

The marked differences between the RA model predictions and those of the RARI and RP models can be primarily attributed to (1) the general lower variance assigned to the Agencies' model inputs and (2) the large difference in estimates for time spent outside the hive and foraging.

There were differences and similarities in the correlation analyses carried out for the RA and RARI models. When negative correlation was assumed between the number of trips $T$ and trip duration $D_{i}$, the RA model predicted predominantly less variability, while the RARI model predicted generally lower estimates of nectar requirement, $D_{\text {nectar }}$ This is likely predominantly due to the switch from assuming trip duration, $D_{i}$, is uniform to assuming that it is lognormal (Table I). Both analyses suggest that when the fraction of trip spent flying, $F_{i}$, is negatively correlated with the number of trips per day, $T$, the correlation has only a small impact on estimated nectar requirement, $D_{\text {nectar }}$ (slightly lower). The fidelity assumption with the RA model resulted in greater variability in $D_{\text {nectar }}$ predictions, whereas with the RARI model 
predictions were generally lower with similar variability. This is also likely related to the switch in distribution from uniform to lognormal for $D_{i}$. When high negative correlations and fidelity were assumed concurrently, both models predicted lower estimates for nectar requirement, $D_{i}$, but the effect was more pronounced with the RARI model. In general, if central point estimates are used in exposure assessment with the Agencies' model, correlations may not be of great concern; however, if probabilistic exposure estimates are to be used, it may be important to consider possible correlations and seek empirical data to support correlation assumptions.

Sensitivity analyses revealed that the most important model inputs in the base cases, in terms of contribution to variance and rank correlation, are sugar required to meet resting metabolic rate, $S_{\mathrm{R}}$, in the RARI model ( $44 \%$ contribution to variance in the base case), and time outside the hive, $T_{\mathrm{O}}$, in the RP model $(49 \%$ contribution to variance in the base case). Notably, in the RARI model, trip duration, $D_{i}$, and number of trips, $T$, contributed a total of $46 \%$ to the variance. Since these variables are the basis for time outside the hive, we can conclude that in both models, time outside the hive contributed most to variance. This is followed by sugar required for rest, $S_{\mathrm{R}}$, and sugar required inside the hive, $S_{\mathrm{I}}$, for the RARI and RP models, respectively. These inputs are relatively important based on contribution to variance and rank correlation. This is likely because bees are presumed to be resting most of the time. $S_{\mathrm{R}}$ was treated as a constant in the Agencies' model and contributed to the much lower variability in the Agencies' predictions.

Other inputs contribute $\leq 11 \%$ to variance in both models' estimates. Results of the elasticity analyses suggest that plausible changes to model input standard deviations, distribution assumptions, and threshold values generally have low impact to model predictions for both the RARI and RP models, with mean and median estimates generally resulting in $\leq 26 \%$ difference from the base case. Changes to mean estimates for input distribution had the greatest impact on model predictions and in some instances changed the order of contribution to variance and ranked correlations of input variables. In the RARI model, trip duration, $D_{i}$, replaced sugar required for rest, $S_{\mathrm{R}}$, for top contributor to variance and top rank correlation when the mean for sugar required for flight, $S_{\mathrm{R}}$, or the mean for trip duration, $D_{i}$, was increased by $50 \%$, and when a $50 \%$ reduction in mean was assumed for sugar required for rest, $S_{\mathrm{R}}$. In the RP model, sugar required inside the hive, $S_{\mathrm{R}}$, took top contributor to variance and top rank correlation when a $50 \%$ lower mean was assumed for sugar required outside the hive, $S_{\mathrm{O}}$, or time spent outside the hive, $T_{\mathrm{O}}$. Thus, sugar required when not foraging may be a more important variable in the models if bees spend less time outside foraging and if the energy difference between foraging and not foraging is lower. The greatest changes to predictions of nectar requirement, $D_{\text {nectar}}$, were seen with $50 \%$ change in the means of sugar content of nectar in both the RARI and RP models with mean and median predictions ranging from 35 to $264 \%$ of the base case predictions. In pesticide risk assessment of specific crops or crop groups, uncertainty could be reduced by using actual nectar sugar content of the treated crop and surrounding vegetation that may have come in contact with the pesticide. Additional data on nectar sugar content of crop plants could be useful to improve representativeness of the Agencies' model for risk assessment in US agroecosystems.

The experiments reported in Dosselli et al. (2016) and Thompson et al. (2016) which were heavily relied on in the generation of time-related input parameters $\left(T, D_{i}\right.$, and $\left.T_{\mathrm{O}}\right)$ were carried out under specific site and environmental conditions in Australia and the UK and may not be representative of colonies foraging in US agroecosystems. Potential disparities, with respect to flight statistics, may stem from differences in climate, available food resources, hive management practices, and colony genetics. Thompson et al. (2016) designed their Lincolnshire study such that the monitored foragers would predominantly forage on oilseed rape grown under typical agricultural conditions. The average temperatures reported in Lincoln during 
the months of the study (May and June 2013) were 11 and $14^{\circ} \mathrm{C}$, which is lower than would be expected over the growing season in the contiguous USA. The Dosselli et al. (2016) study was conducted at the University of Western Australia, which is located in Crawley, near Perth. The area is generally urban with nearby parks and botanical gardens. Also, in neither study was the subspecies reported. Subspecies and strains of Apis mellifera may display subtle differences in metabolism and forage differently (e.g., Harrison and Fewell 2002; Ibrahim et al. 2010). Additional data capturing intra-individual variability from typical Apis mellifera foraging on nectar in US agroecosystems would be useful to confirm that the data used here are suitably representative.

The use of readily available data from the literature, in particular relatively recent RFID observations, enhances predictions of nectar requirements for nectar foragers. These data capture the wide variability expected among individuals. Risk assessors should consider available data relevant to dietary intake when estimating honey bee exposure via the diet.

\section{ACKNOWLEDGMENTS}

Thanks to Dwayne Moore for comprehensive review of earlier versions of this manuscript.

\section{AUTHOR'S CONTRIBUTIONS}

VK conceived the idea, SR carried out the modeling, and VK reviewed and contributed to revisions of it. All authors approved the final manuscript.

\section{FUNDING INFORMATION}

Funding for this effort was provided by the Pollinator Research Task Force.

\section{COMPLIANCE WITH ETHICAL STANDARDS}

Conflict of interest SR declares that she has no potential conflict of interest in relation to the study in this paper. VK declares that his employer, Corteva Agriscience, develops and markets crop protection products that are subject to agency regulatory approvals that include consideration of pollinator risk assessments. Corteva Agriscience is a member of the Pollinator Research Task Force, an international consortium of producers of crop protection products organized for the purpose of improving the science of pollinator risk assessment.

\section{OPEN ACCESS}

This article is distributed under the terms of the Creative Commons Attribution 4.0 International License (http://creativecommons.org/licenses/by/4.0/), which permits unrestricted use, distribution, and reproduction in any medium, provided you give appropriate credit to the original author(s) and the source, provide a link to the Creative Commons license, and indicate if changes were made.

Évaluation probabiliste des besoins en nectar des abeilles qui récoltent le nectar.

probabiliste / modélisation / nectar / Apis mellifera / abeille mellifère / besoin énergétique

Wahrscheinlichkeitsabschätzung der Anforderungen für Nektar bei Nektarsammlerinnen der Honigbiene.

Wahrscheinlichkeit / Modellierung / Nektar / Anforderungen für Nektarsammlerinnen

\section{REFERENCES}

Balderrama, N. M., Almeida, L. O., Núñez, J. A. (1992). Metabolic rate during foraging in the honeybee. J. Comp. Physiol. B. 162, 440-447.

Butler, C. G., Jefree, E. P. Kalmus H. (1943). The behaviour of a population of honeybees on an artificial and on a natural crop. J. Exp. Biol. 20, 65-73.

Cahill, K., Lustik, S. (1976). Oxygen consumption and thermoregulation in Apis mellifera workers and drones. Comp. Biochem. Physiol. 55A, 355-357.

Chen, J.C.P., Chou, C. C. (1993). Cane Sugar Handbook, 12th Edition, A Manual for Cane Sugar Manufacturers and their Chemists. John Wiley \& Sons, Inc., New York, New York. Page 24.

Coelho, J. R., Mitton, J. B. (1988). Oxygen consumption during hovering is associated with genetic variation of enzymes in honey bees. Funct. Ecol. 2(2), 141-146.

Dosselli, R., Grassl, J., Carson, A., Simmons, L. W., Baer, B. (2016). Flight behaviour of honey bee (Apis mellifera) workers is altered by initial infections of 
the fungal parasite Nosema apis. Sci. Rep. 6, 36649; https://doi.org/10.1038/srep36649.

Eckert, C. D., Winston, M. L., Ydenberg, R. C. (1994). The relationship between population size, amount of brood, and individual foraging behaviour in the honey bee, Apis mellifera L . Oecologia 97, 248-255.

EFSA (European Food Safety Authority). 2013. EFSA Guidance Document on the risk assessment of plant protection products on bees (Apis mellifera, Bombus spp. and solitary bees). EFSA J 11(7) 3295.

Fahrenholz, L., Lamprecht, I. Schricker, B. (1989). Microcalorimetric investigations of the energy metabolism of honeybee workers, Apis mellifera carnica. Thermochim. Acta 1951, 13-21.

Feuerbacher, E., Fewell, J. H., Roberts, S. P., Smith, E. F., Harrison, J. F. (2003). Effects of load type (pollen or nectar) and load mass on hovering metabolic rate and mechanical power output in the honey bee Apis mellifera. J. Exp. Biol. 206, 1855-1865.

Goller, F. and Esch, H. E. (1991). Oxygen consumption and flight muscle activity during heating in workers and drones of Apis mellifera. J. Comp. Physiol. B. 161, $61-67$.

Harano, K., Nakamura, J. (2016). Nectar loads as fuel for collecting nectar and pollen in honeybees: adjustment by sugar concentration. J. Comp. Physiol. 202, 435443.

Harrison, J. F., Fewell, J. H. (2002). Environmental and genetic influences on flight metabolic rate in the honey bee, Apis mellifera. Comp. Biochem. Phys. A. 133(2), 323-333.

He, X., Wang, W., Qin, Q., Zeng, Z. Zhang S., Barron A. B. (2013). Assessment of flight activity and homing ability in Asian and European honey be species, Apis cerana and Apis mellifera, measured with radio frequency tags. Apidologie 44, 38-51.

Headrick, T. C. (2010). Statistical Simulation: Power Method Polynomials and other Transformations. Chapman \& Hall/CRC, Boca Raton, FL.

Heberle, J. A. (1914). Notes from Germany: how many trips to the field does a bee make in a day? How long does it take to fetch a load? How long does a bee remain in the hive between trips? Glean. Bee Cult. 42, 904-905.

Heusner, A., Stussi, T. H. (1964). Métabolisme énergétique de l'abeille isolée: Son role dans la thermorégulation de la ruche. Insectes Sociaux. 11(3),239-265.

Ibrahim, C., Song, D., Mixson, T., Serrano, E., Clement, M. et al; (2010). Foraging Response of Turkish Honey Bee Subspecies to Flower Color Choices and Reward Consistency. J. Insect Behav. 23(2), 100-116.

Knopper, L. D., Dan, T., Reisiq, D. D., Johnson, J. D., Bowers, L.M. (2016). Sugar concentration in nectar: a quantitative metric of crop attractiveness for refined pollinator risk assessments. Pest Manag. Sci. 72(10), 1807-1812.

Kovac, H., Stabentheiner, A., Hetz, S. K., Petz, M., Crailsheim, A. (2007). Respiration of resting honey bees. J. Insect Physiol. Behav. 53, 1250-1261.
Lach, L., Kratz, M., Baer B. (2015). Parasitized honey bees are less likely to forage and carry less pollen. J. Invertebr.Pathol. 130, 64-71.

Lundie, A. E. (1925). The Flight Activities of the Honeybee. United States Department of Agriculture, Department Bulletin No. 1328. Washington, DC. May, 1925.

Paleari, L., Confalonieri, R. (2016). Sensitivity analysis of a sensitivity analysis: We are likely overlooking the impact of distribution assumptions. Ecological Modeling. 340, 57-63.

Park, W. (1922). Time and labor factors involved in gathering pollen and nectar. Am Bee J. 42(6), 254-255.

Perry, C. J., Søvik, E., Myerscough, M. R., Barron, A. B. (2015). Rapid behavioral maturation accelerates failure of stressed honey bee colonies. PNAS 113(30), 342734232 .

Ribbands, C. R. (1949). The foraging method of individual honey-bees. J. Anim. Ecol. 18(1), 47-66.

Rodney, S., Purdy, J. This issue. Nectar provisioning and ingestion by honey bee foragers, and colony-level food allocation: A review to support assessment of dietary exposure to pesticides. Apidologie.

Rortais, A., Arnold, G., Halm, M-P., Touffet-Briens, F. (2005). Modes of honeybee exposure to systemic insecticides: estimated amounts of contaminated pollen and nectar consumed by different categories of bees. Apidologie 36(1): 71-83.

Rothe, U., Nachtigall, W. (1989). Flight of the honey bee. J. Comp. Physiol. B. 158:739-749.

Southwick, E. E. (1982). Metabolic energy of intact honey bee colonies. Comp. Biochem. Physiol.. 71A, 227281.

Southwick, E. E. (1983). The honey bee cluster as a homeothermic superorganism. Comp. Biochem. Physiol. 75A(4), 641-645.

Stabentheiner, A., J. Vollmann, H. Kovac and K. Crailsheim. 2003. Oxygen consumption and body temperature of active and resting honeybees. J. Insect Physiol. 49:881-889.

Thom, C., Seeley, T. D. Tautz, J. (2000). A scientific note on the dynamics of labor devoted to nectar foraging in a honey bee colony: number of foragers versus individual foraging activity. Apidologie 31(6), 737-738.

Thompson, H., Coulson, M., Ruddle, N., Wilkins, S. Harkin, S. (2016). Thiamethoxam: assessing flight activity of honeybees foraging on treated oilseed rape using radio frequency identification technology. Environ. Toxicol. Chem. 35(2), 385-393.

Thompson, H., (2018) Personal communication with S. Rodney.

USEPA (US Environmental Protection Agency). (2009). Guidance on the Development, Evaluation, and Application of Environmental Models., Office of the Science Advisor, Council for Regulatory Environmental Modeling, Washington D.C. EPA/100/K-09/003

USEPA (US Environmental Protection Agency), PMRA (Pest Management Regulatory Agency) and CDPR (California Department of Pesticide Regulation). 
(2012). White Paper in Support of the Proposed Risk Assessment Process for Bees. Submitted to the FIFRA Scientific Advisory Panel for Review and Comment, September 11-14, 2012. Office of Chemical Safety and Pollution Prevention (US Environmental Protection Agency), Pest Management Regulatory Agency (PMRA) Health Canada, and California Department of Pesticide Regulation (CDPR). http://www.regulations. gov/\#!documentDetail;D=EPA-HQ-OPP-2012-05430004), Accessed 21 October 2019.

USEPA (US Environmental Protection Agency), PMRA (Pest Management Regulatory Agency) and CDPR (California Department of Pesticide Regulation). (2014). Guidance for Assessing Pesticide Risks to Bees. Office of Pesticide Programs, US Environmental Protection Agency, Washington, DC, Health Canada Pest Management Regulatory Agency, Ottawa, ON, and California Department of Pesticide Regulation, Sacramento,
CA. https://www.epa.gov/sites/production/files/2014-06 /documents/pollinator_risk assessment_guidance 0619 14.pdf, Accessed 21 October 2019.

Vose, D. (1996). Quantitative risk analysis: A guide to Monte Carlo simulation modeling. John Wiley and Sons. Chichester, UK. 328 pp.

Wolf, T. J., Schmid-Hempel, P., Ellington, C. P., Stevenson, R. D. (1989). Physiological correlates of foraging efforts in honey-bees: Oxygen consumption and nectar load. Funct. Ecol. 3(4), 417-424.

Publisher's note Springer Nature remains neutral with regard to jurisdictional claims in published maps and institutional affiliations. 